\title{
Extração de nutrientes pela parte aérea de carqueja sob a influência de fontes e doses de nitrogênio
}

\author{
Nutrient extraction for shoots of carqueja under fonts and levels of nitrogen \\ Angela Maria Palacio Cortés ${ }^{\mathrm{I}}$ Luiz Antonio Biasi ${ }^{\mathrm{II}}$ Beatriz Monte Serrat ${ }^{\mathrm{II}}$ \\ Tomoe Nakashima ${ }^{\text {IV }}$
}

\begin{abstract}
- NOTA -
RESUMO

O objetivo deste trabalho foi determinar a influência das fontes e doses de nitrogênio no teor e na extração de nutrientes na parte aérea de carqueja seis meses após o plantio, assim como sua relação com o rendimento de matéria seca e óleo essencial. O experimento foi conduzido na Estação Experimental de Canguiri (UFPR), com delineamento em blocos ao acaso, com sete tratamentos e quatro repetições em parcelas de $2 \mathrm{~m}^{2}$. Os tratamentos foram 4, 8 e $16 \mathrm{~g}$ de nitrogênio $(N)$ por planta, na forma de uréia; as mesmas quantidades de

(UFPR), with experimental design in random blocks with seven treatments and four repetitions. The treatments consisted of 4 , 8 and 16 grams of nitrogen $(N)$ per plant of urea and manure sources, and a control without $N$ application. The fertilization was done two times with an interval of two months. There was no statistical difference between content and extraction of nutrients for treatments for these soil fertility conditions. Similar results were observed for dry matter and essential oil production. The nutrient extraction for aerial parts presented the decreasing sequence $\mathrm{K}>\mathrm{N}>\mathrm{Ca}>\mathrm{S}>\mathrm{Mg}>\mathrm{P}$ for macronutrients and $\mathrm{Mn}$ $>\mathrm{Fe}>\mathrm{B}>\mathrm{Zn}>\mathrm{Cu}$ for micronutrients.
\end{abstract} $N$ na forma de esterco ovino; e o controle, sem aplicação de $N$. As adubações realizaram-se em duas aplicações com intervalo de dois meses. Não foram verificadas diferenças estatisticamente significativas entre tratamentos para o teor e a extração de nutrientes nestas condições de fertilidade do solo, o que também foi observado para a produção de matéria seca e o teor de óleo essencial. A quantidade de nutrientes extraídos pela parte aérea apresenta a seqüência decrescente $\mathrm{K}>\mathrm{N}>\mathrm{Ca}>\mathrm{S}>\mathrm{Mg}$ $>$ P para os macronutrientes e de $\mathrm{Mn}>\mathrm{Fe}>\mathrm{B}>\mathrm{Zn}>\mathrm{Cu}$ para os micronutrientes.

Palavras-chave: Baccharis trimera, adubação nitrogenada, macronutrientes, micronutrientes.

\section{ABSTRACT}

This research was aimed at determining the influence of nitrogen sources and levels on the amount and concentration of nutrients extracted from the shoots of carqueja six months after planted. The amount and concentration were correlated to the essential oil and dry matter production. The experiment was conducted at Experimental Station of Canguiri
Key words: Baccharis trimera, nitrogen fertilization, macronutrients, micronutrients.

Baccharis trimera (Less) DC, popularmente conhecida como carqueja, carqueja-amarga, bacanta ou vassoura (LORENZI \& MATOS, 2002), é uma espécie usada na medicina popular por suas atividades antiinflamatória, analgésica e antiulceral (CASTRO, 1996; CASTRO \& FERREIRA, 2000). Além disso, também é utilizada na indústria de cervejaria como substituto do lúpulo e na aromatização de refrigerantes e licores (CASTRO \& FERREIRA, 2000). Seus hábitos de crescimento são de elevada rusticidade, sendo encontrada em solos pobres e ácidos, em lugares úmidos nas ribanceiras dos rios e até $2.800 \mathrm{~m}$ de altitude (CORREA JUNIOR et al., 1994), preferindo condições

\footnotetext{
IPrograma de Pós-graduação em Agronomia, Universidade Federal de Paraná (UFPR), Curitiba, PR, Brasil. E-mail: a_mariap@yahoo.com.br.

IIDepartamento de Fitotecnia e Fitossanitarismo, UFPR, CP 19061, 81531-990, Curitiba, PR, Brasil. E-mail: biasi@ufpr.br. Autor para correspondência.

IIIDepartamento de Solos, UFPR, Curitiba, PR, Brasil.

${ }^{\text {IV }}$ Departamento de Farmácia, UFPR, Curitiba, PR, Brasil.
} 
de pleno sol para crescer (BONA, 2002). Existem poucas informações dos aspectos nutricionais de plantas nativas e de seu desenvolvimento em ambientes cultivados, sendo necessária a definição de padrões de comportamento para a otimização da produção e dos rendimentos futuros, principalmente em áreas olerícolas, as quais recebem elevadas quantidades de adubos, principalmente nitrogenados, podendo ocorrer alteração do estado nutricional das plantas.

O objetivo deste trabalho foi quantificar o teor e a extração de macro e micronutrientes pela parte aérea de carqueja seis meses após o plantio, sendo esta submetida a doses crescentes de nitrogênio, considerando-se a uréia como fonte de fertilizante mineral e o esterco ovino como fonte de fertilizante orgânico. Além disso, procurou-se determinar a correlação do teor de nutrientes da parte aérea com a produção de matéria seca e o teor de óleo essencial produzido.

O experimento foi conduzido na estação Experimental do Canguiri (Pinhais-PR), num solo com: P (resina): 81mg dm${ }^{-3}$; M.O: $78 \mathrm{~g} \mathrm{dm}^{-3} ; \mathrm{K}: 0,63 \mathrm{cmol}_{\mathrm{c}} \mathrm{dm}^{-3}$; Ca: 7,6 $\mathrm{cmol}_{\mathrm{c}} \mathrm{dm}^{-3}$. Mg: 2,8 $\mathrm{cmol}_{\mathrm{C}} \mathrm{dm}^{-3}$; SB: $11,03 \mathrm{cmol}_{\mathrm{c}}$ $\mathrm{dm}^{-3}$; CTC: $18,23 \mathrm{cmol}_{\mathrm{c}} \mathrm{dm}^{-3} ; \mathrm{V}: 61 \%$, saturação de $\mathrm{Al}$ : 1,8\% e pH $\left(\mathrm{CaCl}_{2}\right): 5,1$, valores determinados pelo Laboratório de Solos e Plantas da Fundação ABC. O plantio foi realizado no mês de maio de 2003, com mudas formadas a partir de estacas de $30 \mathrm{~cm}$ de comprimento, coletadas de plantas masculinas da coleção de plantas medicinais da Estação Experimental do Canguiri. Essas mudas foram mantidas em caixas plásticas com fundo perfurado e substrato de casca de arroz carbonizada, sob irrigação intermitente, por um período de 70 dias, para seu enraizamento, na casa-de-vegetação do Departamento de Fitotecnia e Fitossanitarismo do Setor de Ciências Agrárias - UFPR. A exsicata da espécie número 45093 foi incorporada ao Herbário do Setor de Ciências Biológicas - UFPR.

O delineamento experimental foi em blocos ao acaso com 4 repetições, em parcelas de $1 \mathrm{~m} \times 2 \mathrm{~m}$ com 8 plantas, com espaçamento entre plantas de $0,5 \mathrm{~m}$ $\mathrm{x} 0,5 \mathrm{~m}$, em linhas duplas com espaço de $1 \mathrm{~m}$ (equivalente a 26.660 plantas por hectare), com um total de 28 parcelas. Foram avaliados sete tratamentos organizados em duas fontes: uréia e esterco ovino, e três doses de nitrogênio por planta: 4, 8, 16 gramas (equivalentes a 106, 213 e 426kg de N por hectare). O tratamento controle não recebeu adubação nitrogenada. Os tratamentos foram colocados mantendo-se uma distância de pelo menos $10 \mathrm{~cm}$ do tronco, ao redor das plantas, em forma circular. A adubação foi fracionada nos meses de agosto e outubro de 2003, determinando-se previamente o conteúdo de nitrogênio e a percentagem de umidade do esterco ovino, considerando-se uma eficiência de liberação de 50\% (COMISSÃO DE FERTILIDADE DO SOLO - RS/SC, 1994). Os teores de nutrientes encontrados na análise do esterco foram: $25 \mathrm{~g} \mathrm{~kg}^{-1} \mathrm{de}$ $\mathrm{N} ; 25 \mathrm{~g} \mathrm{~kg}^{-1}$ de $\mathrm{P}_{2} \mathrm{O}_{5} ; 21 \mathrm{~g} \mathrm{~kg}^{-1}$ de $\mathrm{K}_{2} \mathrm{O} ; 7$, $1 \mathrm{~g} \mathrm{~kg}^{-1}$ de $\mathrm{Mg}$; 15,3g kg-1 de Ca; 3,06g $\mathrm{kg}^{-1}$ de $\mathrm{Na}$; e, para os micronutrientes: 426mg kg-1 de Zn; $21 \mathrm{mg} \mathrm{kg}^{-1}$ de $\mathrm{Cu}$; $143 \mathrm{mg} \mathrm{kg}^{-1}$ de $\mathrm{Mn}$; $906 \mathrm{mg} \mathrm{kg}^{-1}$ de Fe; resíduo mineral de $17,7 \%$, densidade de $0,24 \mathrm{~g} \mathrm{~cm}^{-3}$ e matéria seca de $42,08 \%$.

A colheita foi realizada seis meses após o plantio, no estado vegetativo. Retirou-se toda a parte aérea, deixando-se $30 \mathrm{~cm}$ de caule para rebrote; a biomassa seca foi determinada 15 dias após secagem, em condições de sombra, até alcançar 7\% de umidade. O óleo essencial foi extraído através do método da hidrodestilação por arraste de vapor d’água, utilizandose o aparelho de Clevenger (GUENTHER, 1972), no Laboratório de Fitoquímica do Departamento de Farmácia - UFPR. Para tal usaram-se 100 gramas da parte aérea da planta, durante um período de quatro horas. A análise do tecido foliar de amostras secas de cada tratamento foi feita pelo Laboratório de Solos e Plantas da Fundação ABC pelas metodologias de digestão nitro-perclórica para $\mathrm{P}, \mathrm{K}, \mathrm{Ca}, \mathrm{Mg}, \mathrm{S}, \mathrm{Fe}$, $\mathrm{Cu}, \mathrm{Zn}, \mathrm{B}$ e Mn, de digestão sulfúrica para o N-total e de incineração (digestão por via seca) para o B (MALAVOLTA et al., 1997). A extração de macronutrientes em $\mathrm{kg} \mathrm{ha}^{-1}$ e de micronutrientes em $\mathrm{g}$ ha ${ }^{-1}$ pela parte aérea foi obtida a partir da sua biomassa produzida por tratamento (extrapolada para hectare) e do teor de nutrientes nela encontrados. As análises de variância foram realizadas pelo teste $\mathrm{F}$ em nível de 5 e $1 \%$, usando-se o programa MSTATC.

A produção de matéria seca e o teor de óleo essencial não apresentaram diferenças estatísticas significativas em relação às fontes e doses de nitrogênio aplicadas, indicando que a cultura de carqueja em solos com altos teores de matéria orgânica poderá ser conduzida aproveitando o $\mathrm{N}$ disponível para a primeira fase de desenvolvimento. O rendimento médio de matéria seca para os sete tratamentos foi de 144,95g planta $^{-1}$, equivalente a 3,86t ha-1, valor superior ao reportado por DAVIES (1999), que obteve $62 \mathrm{~g} \mathrm{planta}^{-1}$ 12 meses após o plantio. Assim, esse autor sugeriu que a primeira colheita fosse feita mais cedo para que se aproveitasse maior número de cortes, gerando, conseqüentemente, maior fonte de renda ao produtor.

A produção média de óleo essencial foi de $16 \mathrm{~mL} \mathrm{~kg}^{-1}$ de planta seca, equivalente a $64 \mathrm{~L} \mathrm{ha}^{-1}$, sendo maior do que os rendimentos obtidos por SIQUEIRA et al. (1985) e por SOUSA et al. (1991), em plantas coletadas em período de floração, os quais variaram 
entre 3 e $10 \mathrm{~mL} \mathrm{~kg}^{-1}$. No entanto, SIMIONATTO et al. (2003) e BONA (2002) acharam teores próximos aos obtidos neste experimento, sendo o valor máximo de $15 \mathrm{~mL} \mathrm{~kg}^{-1}$.

As análises de variância do teor de macro e micronutrientes das partes aéreas da carqueja não revelaram diferenças estatísticas significativas (Tabela 1), evidenciando que não houve influência das doses de nitrogênio aplicadas e nem mesmo dos outros elementos aportados no esterco ovino. Possivelmente, o alto nível de fertilidade do solo não permitiu respostas marcantes das plantas à adição de nutrientes, podendo ser que elas estivessem no ponto de máximo metabolismo e absorção de nutrientes, sem serem alterados o teor de óleo essencial e a produção de matéria seca. Caso essas doses fossem aumentadas, a planta poderia passar a um ponto crítico em que o adubo, além de não conseguir aumentar a produtividade, ocasionaria sintomas de excesso nas plantas, fato não observado neste experimento.

De acordo com a média geral dos tratamentos, o teor de nutrientes na parte aérea da carqueja apresentou a seguinte ordem: $\mathrm{K}>\mathrm{N}>\mathrm{Ca}>\mathrm{S}$ $>\mathrm{Mg}>\mathrm{P}$ para os macronutrientes, e $\mathrm{Mn}>\mathrm{Fe}>\mathrm{B}>\mathrm{Zn}$ $>$ Cu para os micronutrientes.

A extração de nutrientes pela parte aérea de carqueja não revelou diferenças estatísticas significativas na análise de variância, demonstrando que não houve influência dos tratamentos aplicados. Mesmo a maior quantidade de adubo ministrada não representou uma maior extração de nutrientes, indicando que, com o nível de fertilidade inicial do solo, já haviam sido atingidas as necessidades de $\mathrm{N}$ e K, com excedente para os demais nutrientes, exceto para Mn, pela sua elevada extração.
Para cada hectare de parte aérea colhida, seis meses após o plantio, espera-se uma extração média de macronutrientes em kg ha-1 de: K: 94; N: 66; Ca: 19; S: 7; P: 7 e Mg: 7, e de micronutrientes em g ha-1 de: Mn: 1.086; Fe: 628; Zn: 117; B: 117 e Cu: 35. Esta seqüência de absorção não foi influenciada pela quantidade de biomassa produzida.

Para plantas de carqueja cultivadas em solo com nível alto de fertilidade, o teor e a extração de nutrientes, a matéria seca e o teor de óleo essencial são independentes da dose e do tipo de adubação nitrogenada testada.

\section{AGRADECIMENTOS}

À Coordenação de Aperfeiçoamento de pessoal de Nível Superior (CAPES), pela concessão de bolsa de mestrado a Cortés; ao Conselho Nacional de Desenvolvimento Científico e tecnológico (CNPq), pela concessão de bolsa de Produtividade em Pesquisa a Biasi.

\section{REFERÊNCIAS}

BONA, C.M. de. Estaquia, calagem e sombreamento de carqueja. 2002. 80f. Dissertação (Mestrado em Agronomia) - Programa de Pós-graduação em Agronomia - Produção Vegetal, Setor de Ciências Agrárias, Universidade Federal do Paraná.

CASTRO, H.G. Estúdio anatômico da carqueja (Baccharis myriocephala D.C.). Viçosa: UFV, 1996. 21p.

CASTRO, H.G.; FERREIRA, F.A. Contribuição ao estudo das plantas medicinais CARQUEJA (Baccharis genistelloides). Viçosa: UFV, 2000. 102p.

COMISSÃO DE FERTILIDADE DO SOLO - RS/SC. Recomendações de adubação e de calagem para os estados do Rio Grande do Sul e Santa Catarina. 3.ed. Passo Fundo: SBCS Núcleo Regional Sul, 1994. 224p.

Tabela 1 - Teor de nutrientes da parte aérea de carquejas, seis meses após plantio, sob influência de doses de uréia e esterco ovino, sendo que os teores 4, 8 e 16 gramas de $\mathrm{N}$ dos tratamentos representam 106, 213 e 426kg ha' ${ }^{-1}$. Pinhais-PR. 2003.

\begin{tabular}{|c|c|c|c|c|c|c|c|c|c|c|c|c|}
\hline \multicolumn{2}{|c|}{ Tratamentos } & \multicolumn{11}{|c|}{ Elementos } \\
\hline \multirow[t]{2}{*}{ Fase } & $\begin{array}{c}\text { Dose N } \\
\left(\text { g planta }^{-1}\right)\end{array}$ & $\mathrm{N}$ & $\mathrm{P}$ & $\mathrm{K}$ & $\mathrm{Ca}$ & $\mathrm{Mg}$ & $\mathrm{S}$ & $\mathrm{Fe}$ & $\mathrm{Cu}$ & $\mathrm{Zn}$ & B & Mn \\
\hline & & \multicolumn{5}{|c|}{ 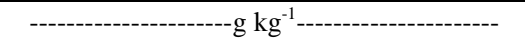 } & \multicolumn{6}{|c|}{ 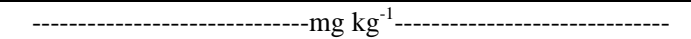 } \\
\hline Controle & 0 & $17,18^{\mathrm{ns}}$ & $1,78^{\mathrm{ns}}$ & $24,80^{\mathrm{ns}}$ & $4,65^{\mathrm{ns}}$ & $1,83^{\text {ns }}$ & $1,73^{\mathrm{ns}}$ & $199,03^{\text {ns }}$ & $9,13^{\text {ns }}$ & $30,80^{\mathrm{ns}}$ & $33,90^{\mathrm{ns}}$ & $295,0^{\text {ns }}$ \\
\hline Uréia & 4 & 16,53 & 1,58 & 22,95 & 4,53 & 1,83 & 1,73 & 155,05 & 8,43 & 26,60 & 31,13 & 268,0 \\
\hline Uréia & 8 & 15,72 & 1,68 & 23,33 & 4,55 & 1,45 & 2,40 & 132,33 & 9,68 & 31,88 & 26,70 & 219,8 \\
\hline Uréia & 16 & 17,95 & 1,70 & 24,35 & 4,90 & 2,10 & 1,83 & 215,60 & 9,13 & 27,25 & 30,98 & 328,5 \\
\hline Esterco & 4 & 17,95 & 1,93 & 25,08 & 5,53 & 1,85 & 1,78 & 182,83 & 9,38 & 30,65 & 31,38 & 282,7 \\
\hline Esterco & 8 & 17,65 & 1,85 & 25,63 & 5,33 & 1,70 & 1,68 & 134,48 & 8,95 & 33,87 & 28,53 & 293,0 \\
\hline Esterco & 16 & 17,43 & 1,73 & 24,18 & 5,05 & 1,85 & 1,63 & 138,43 & 8,80 & 29,13 & 31,65 & 296,1 \\
\hline CV (\%) & & 13,40 & 7,98 & 5,09 & 10,36 & 21,18 & 29,38 & 23,37 & 9,37 & 28,35 & 17,71 & 24,04 \\
\hline
\end{tabular}

${ }^{\text {ns }}$ Não-significativo em nível de 5\%. 
CORREA JÚNIOR, C. et al. Cultivo de plantas medicinais, condimentares e aromáticas. Jaboticabal: FUNEP, 1994. 151p.

DAVIES, P. Experimentation on the propagation of Baccharis trimera (Less.) D.C. Compositae (CARQUEJA). Acta Horticulturae, Belgium, n.502, p.117-120, 1999.

GUENTHER, E. The essential oils. New York: D. Van Nostrand, 1972. V.1, p.316-319.

LORENZI, H.; MATOS, F.J.A. Plantas medicinais no Brasil: nativas e exóticas cultivadas. Nova Odessa: Instituto Plantarum, 2002. p.141-142.

MALAVOLTA, E. et al. Avaliação do estado nutricional das plantas. Piracicaba: Associação Brasileira para Pesquisa da Potassa e do Fosfato, 1997. 310p.
SIMIONATTO, E. et al. Analise química e atividade antimicrobiana dos óleos voláteis de Baccharis trimera e Baccharis articulata do Rio Grande do Sul. In: SIMPÓSIO BRASILEIRO DE ÓLEOS ESSENCIAIS, 3., 2003, Campinas. Anais... Campinas (SP): Instituto Agronômico (IAC), 2003. p.74.

SIQUEIRA, N.C.S. et al. Análise comparativa dos óleos essenciais de Baccharis articulata (Lam) Pers. e Baccharis trimera (Less.) DC. (Compositae), espécies espontâneas no Rio Grande do Sul. Revista Brasileira de Farmácia, Rio de Janeiro, n.3, p.36-39, 1985.

SOUSA, M.P. et al. Constituintes químicos ativos de plantas medicinais brasileiras. Fortaleza: UFC, 1991. 416p. 